5. A. B. Paalman-de Miranda, Topological semigroups, Mathematical Centre Tracts, Amsterdam, 1964.

6. A. D. Wallace, The Rees-Suschkewitsch structure theorem for compact simple semigroups, Proc. Nat. Acad. Sci. U.S.A. 42 (1956), 430-432.

7. - Relative ideals. II, Acta Math. 14 (1963), 137-148.

8. - - Retractions in semigroups, Pacific J. Math. 7 (1957), 1513-1517.

UNIVERSITY OF FLORIDA AND

UNiversity OF MASSACHUSETtS

\title{
AN EXAMPLE OF TWO UNIFORMITIES EQUAL IN HEIGHT AND PROXIMITY
}

JACK L. HURSCH, JR.

1. Introduction. In [7] Yu. M. Smirnov first raised the question whether there existed a proximity class without a largest member. The first example was given in [2] and later examples occurred in [4] and [6].

In [5] the author introduced the concept of height of uniformities and showed that if two uniformities in the same proximity class were comparable in height but not comparable in the usual ordering, then their least upper bound was not in that proximity class. An example was given. This example was new in the sense that all previous examples had involved pairs of uniformities which were not comparable in height.

The question was also raised whether two uniformities exist which are equal in both proximity and height. This question is answered by the example given in this paper.

In $\$ 2$ we review what we need of proximity and height. In $\$ 3$ we construct the subbasic covers which are used in $\$ 4$ to complete the construction of the two uniformities.

2. Proximity and height. The concept of proximity was first introduced by Efremovič in [3] and more about it occurs in [1], [2], and [6]. We say a uniformity $\mathcal{U}$ is $\leqq^{p}$ to a uniformity $v$ if, for any set $A$ in $X$ and any $U$ in $\mathcal{U}$, there exists $V$ in $v$ such that $\operatorname{St}(A, V) \subset \operatorname{St}(A, U)$, where $\operatorname{St}(A, U)$ stands for star of $A$ with respect to $U$. If both

Presented to the Society, September 1, 1966; received by the editors July 12, 1966. 
$\mathcal{U} \leqq p ~ V$ and $\mathcal{V} \leqq p$, then we say $\mathcal{u}$ and $\mathcal{V}$ are in the same proximity class $\left(=^{p}\right)$. For our purposes, the facts needed about proximity are as follows:

2.1. Each proximity class has a unique, smallest, totally bounded member [1].

We let $\mathcal{u}_{u}$ denote the smallest member of the proximity class of $\mathcal{u}$. If $\mathcal{u}$ and $v$ are two uniformities, then it follows from a lemma in [2] that

2.2. $\left(\mathcal{u} \vee \mathcal{V}_{u}\right)=p\left(\mathcal{u}_{u} \vee \mathcal{V}\right)$ where $\vee$ denotes least upper bound.

Height was first defined in [5]. If $\mathcal{U}$ and $v$ are two uniformities on the same set $X$, then we say $\mathcal{u} \leqq^{h} v$ if, for each $U \in \mathcal{U}$ there exists $V \in \mathcal{V}$ and a finite cover $W$ of $X$ such that $V \wedge W$ is a refinement of $U$, where $\wedge$ stands for intersection as in $[6$, p. 4].

From [5] we need

2.3. If $\mathcal{u}$ is a uniformity and $v$ is a totally bounded uniformity, then $v \leqq h$.

2.4. If $\mathcal{U} \leqq^{h} \mathcal{V}$ then $(\mathcal{U} \vee \mathcal{V})=^{h} \mathcal{V}$.

2.5. If $\mathcal{u}=p \mathcal{V}=p(\mathcal{U} \vee \mathcal{V})$ and $\mathcal{u}{ }^{h} \mathcal{V}$, then $\mathcal{U} \leqq \mathcal{V}$.

2.6. If $u$ has a base consisting of partitions, then $\mathcal{u}_{u}$ has a base consisting of partitions, $W=\left\{B_{\alpha}\right\}, \alpha=1,2, \cdots, n$, where each $B_{\alpha}$ is a union of members of some member of the base for $\mathcal{u}$.

Number 2.6 is a slight generalization of a result appearing in [1, p. 354].

3. Construction of the subbasic covers. Assume $X$ is a countable set ordered like the in tegers. Let any subset of $X$ be ordered according to this original ordering. Let $f$ be a one-to-one map of the integers on to the set of all pairs of integers $(i, j)$ where $i<j$, and let $\pi_{1}$ and $\pi_{2}$ be the projections, $\pi_{1}((i, j))=i, \pi_{2}((i, j))=j$ respectively. Let $\sigma_{1}$ and $\sigma_{2}$ be functions on totally ordered infinite subsets of $X$ defined as follows:

$$
\begin{aligned}
& \sigma_{1}\left(\left\{x_{1}, x_{2}, \cdots\right\}\right)=\left\{x_{1}, x_{3}, x_{5}, \cdots\right\}, \\
& \sigma_{2}\left(\left\{x_{1}, x_{2}, \cdots\right\}\right)=\left\{x_{2}, x_{4}, x_{6}, \cdots\right\} .
\end{aligned}
$$

Let $\sigma_{i}^{k}$ denote the application of $\sigma_{i} k$ times.

We will now inductively construct an infinite number of covers, $a_{i}, i=1,2, \cdots$, of $X$. First, let $a_{1}=\left\{A_{k}^{1}\right\}, k=1,2, \cdots$ where $A_{\mathbf{k}}^{1}=\sigma_{1}\left(\sigma_{2}^{k-1}(X)\right)$. Assume we have constructed $Q_{1}, \cdots, Q_{n-1}$ where $Q_{i}=\left\{A_{k}^{i}\right\}$, and each cover is a partition of $X$. Also assume that each $A_{\mathbf{k}}^{i}$ is the union of $2^{i-1}$ disjoint, infinite sets of the form

3.1. $A_{k_{i}}^{i} \cap A_{k_{i-1}}^{i-1} \cap \cdots \cap A_{k_{1}}^{1} k_{r}$ being a member of $f\left(k_{r+1}\right)$ for $r=1,2, \cdots, i-1$. 
Now, if $l_{1}=\pi_{1}(f(k))$ and $l_{2}=\pi_{2}(f(k))$, we wish to construct $A_{\boldsymbol{k}}^{n}$ so that it intersects each of the $2^{n-2}$ sets of $A_{l_{1}}^{n-1}$ and each of the $2^{n-2}$ sets of $A_{l_{2}}^{n-1}$ in such a way that the $2^{n-1}$ resulting sets satisfy the induction hypothesis. Let $m_{i}$ be the number of times that $l_{i}$ occurs as a member of $f(j)$ for $j<k$.

Then let $A_{\boldsymbol{k}}^{n}$ be the union of the $2^{n-1}$ sets of the form

$$
\sigma_{1}\left(\sigma_{2}^{m_{1}}\left(A_{l_{2}}^{n-1} \cdots\right)\right) \text {. }
$$

It is easy to see that these sets satisfy the induction hypothesis and that the sets $A_{\boldsymbol{k}}^{n}$ form a partition-cover of $X$.

Let $W^{k}$ be a member of the fundamental system of covers for $W_{u}^{k}$ where $\mathscr{W}^{k}$ is the uniformity generated by $\mathfrak{a}_{k}$; then we have:

LEMMA 3.2. For any $n$ and $m<n$,

3.3. $a_{n} \wedge a_{n-1} \wedge \cdots \wedge a_{m+1} \wedge W^{m} \wedge a_{m-1} \wedge \cdots \wedge a_{1}$ does not refine $Q_{m}$.

Proof. By reference to 3.1 we see that a set of 3.3 must be of the form

3.4. $A_{\boldsymbol{k}_{n}}^{n} \cap \cdots \cap A_{\boldsymbol{k}_{m+1}}^{m+1} \cap B_{\alpha} \cap A_{\boldsymbol{k}_{m-1}}^{m-1} \cap \cdots \cap A_{\boldsymbol{k}_{1}}^{1}$, where $B_{\alpha} \in W^{m}$.

Now, if it should happen that

3.5. $k_{m-1}$ is a member of both $f\left(\pi_{1}\left(f\left(k_{m+1}\right)\right)\right)$ and $f\left(\pi_{2}\left(f\left(k_{m+1}\right)\right)\right)$ and

3.6. $B_{\alpha}$ contains both $A_{\pi_{1}}^{m}\left(f\left(k_{m+1}\right)\right)$ and $A_{\pi_{2}}^{m}\left(f\left(k_{m+1}\right)\right)$, then 3.2 cannot refine $a_{m}$.

Let $k_{m-1}=1$, for example. Then if $s_{1}<s_{2}$ are any two integers such that 1 is a member of both $f\left(s_{1}\right)$ and $f\left(s_{2}\right)$; and $s_{3}$ is such that $f\left(s_{3}\right)$ $=\left(s_{1}, s_{2}\right)$, then we have 3.5 , when $k_{m+1}=s_{3}$ and $k_{m-1}=1$. Clearly, no $B_{\alpha} \in W$ can contain both $A_{s_{1}}^{m}$ and $A_{s_{2}}^{m}$, or we would have both 3.5 and 3.6. But there are an infinite number of integers $s$ such that $f(s)$ contains 1 . Thus, since $W^{m}$ has only a finite number of members, 3.5 and 3.6 must occur for some sets in 3.3. Thus, 3.3 cannot refine $Q_{m}$.

Lemma 3.7. For every $k=1,2, \cdots$, there exists a two member cover $\beta=\left\{B_{1}, B_{2}\right\}$ of $X$ such that $Q_{k+1} \wedge \beta$ refines $a_{k}$.

Proof. Let

$$
B_{i}=\bigcup_{j=1}^{\infty} A_{j}^{k+1} \cap A_{\pi_{i}(f(j)),}^{k} \quad i=1,2 .
$$

4. Construction of the two uniformities. Let $u^{\prime}$ be generated by the odd numbered $a_{i}$ 's and let $\sigma^{\prime}$ be generated by the even numbered $a_{i}$ 's. Then $\mathcal{u}^{\prime}={ }^{h} \mathcal{V}^{\prime}$ is a corollary of Lemma 3.7. Let $\mathcal{U}=\mathcal{U}^{\prime} \vee \mathcal{V}_{u}^{\prime}$, and $v=v^{\prime} \vee \mathcal{u}_{u}^{\prime}$. By 2.2, $\mathcal{u}=\boldsymbol{p} v$. By 2.3 and $2.4, \mathfrak{u}={ }^{h} v$. That 
$\mathcal{u} \neq v$ may be seen as follows: Let $a_{m}$ be any member of the generating set for $\mathcal{u}^{\prime}$. Now, any member of $v$ must be refined by a cover of the form of 3.3 which, by Lemma 3.2, cannot refine $a_{m}$. Thus $a_{m} \notin V$.

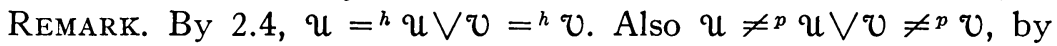
2.5. On the other hand, since every proximity class has a smallest

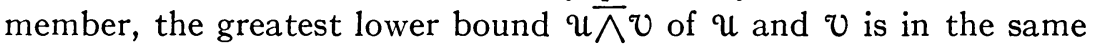
proximity class as both $\mathcal{U}$ and $\mathcal{V}$.

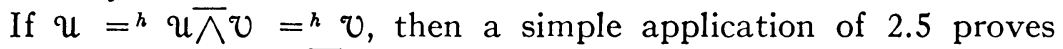
$u=v$. Thus, $u \neq^{h} u \bar{\wedge} v \neq^{h} v$.

\section{REFERENCES}

1. E. M. Alfsen and J. E. Fenstad, On the equivalence between proximity structures and totally bounded uniform structures, Math. Scand. 7 (1959), 353-360.

2. E. M. Alfsen and O. Njåstad (Oslo), Proximity and generalized uniformity, Fund. Math. 52 (1963), 235-252.

3. V. A. Efremovič, Infinitesimal spaces, Dokl. Akad. Nauk SSSR 76 (1951), 341-343. (Russian)

4. J. E. Fenstad, On l-groups of uniformly continuous functions. III, Math. Z. 83 (1964), 133-139.

5. J. L. Hursch, Jr., Proximity and height, Math. Scand. 17 (1965), 150-160.

6. J. R. Isbell, Uniform spaces, Math. Surveys No. 12, Amer. Math. Soc., Providence, R. I., 1964.

7. Y. M. Smirnov, On proximity spaces, Mat. Sb. 31(73) (1952), 543-574; Amer. Math. Soc. Transl. (2) 38 (1964), 5-35.

UNIVERSITY OF VERMONT 